\title{
Effects of immobilization and remobilization on the ankle joint in Wistar rats
}

\author{
R.I. Kunz ${ }^{1}$, J.G. Coradini ${ }^{2}$, L.I. Silva ${ }^{2}$, G.R.F. Bertolini' ${ }^{2}$, R.M.C. Brancalhão ${ }^{1}$ and L.F.C. Ribeiro ${ }^{1}$ \\ ${ }^{1}$ Laboratório de Biologia Estrutural e Funcional, Universidade Estadual do Oeste do Paraná, Cascavel, PR, Brasil \\ ${ }^{2}$ Laboratório do Estudo das Lesões e Recursos Fisioterapêuticos, Universidade Estadual do Oeste do Paraná, \\ Cascavel, PR, Brasil
}

\begin{abstract}
A sprained ankle is a common musculoskeletal sports injury and it is often treated by immobilization of the joint. Despite the beneficial effects of this therapeutic measure, the high prevalence of residual symptoms affects the quality of life, and remobilization of the joint can reverse this situation. The aim of this study was to analyze the effects of immobilization and remobilization on the ankle joint of Wistar rats. Eighteen male rats had their right hindlimb immobilized for 15 days, and were divided into the following groups: G1, immobilized; G2, remobilized freely for 14 days; and G3, remobilized by swimming and jumping in water for 14 days, performed on alternate days, with progression of time and a series of exercises. The contralateral limb was the control. After the experimental period, the ankle joints were processed for microscopic analysis. Histomorphometry did not show any significant differences between the control and immobilized/remobilized groups and members, in terms of number of chondrocytes and thickness of the articular cartilage of the tibia and talus. Morphological analysis of animals from $\mathrm{G} 1$ showed significant degenerative lesions in the talus, such as exposure of the subchondral bone, flocculation, and cracks between the anterior and mid-regions of the articular cartilage and the synovial membrane. Remobilization by therapeutic exercise in water led to recovery in the articular cartilage and synovial membrane of the ankle joint when compared with free remobilization, and it was shown to be an effective therapeutic measure in the recovery of the ankle joint.
\end{abstract}

Key words: Synovial joint; Remobilization in aquatic environment; Morphology; Articular cartilage; Synovial membrane

\section{Introduction}

A sprained ankle is the most common musculoskeletal sports injury (1), and it is estimated that one occurs each day per 10,000 people (2), with a peak incidence between 20 and 30 years of age, predominantly in men (3). Several studies have confirmed a high percentage of individuals who suffer from residual pain, ankle instability, and recurrent sprains (4-6), which raises the cost of health care $(4,7)$.

Despite the different methods used in the treatment of ankle sprains, such as functional, surgical, and conservative measures with fixed or semi-rigid immobilization $(5,8)$, there is no consensus among the cited authors about the best form of treatment $(4,5)$. However, joint immobilization is still the most widely used therapeutic modality (9) and, according to Belangero et al. (4), it is used in over $45 \%$ of partial ligament injuries, and more than $60 \%$ in total ligament injuries.

According to Lamb et al. (10), the immobilization of damaged synovial joints reduces pain when compared to other therapeutic treatments. However, when it is performed in the knee joint, this may adversely affect bone and muscle composition, the blood supply of the immobilized segment, and even joint structure $(11,12)$. According to Widuchowski et al. (13), immobilization can cause and/or worsen chondral lesions, leading, in the long term, to the development of osteoarthritis. Bedi et al. (14) stated that these injuries, associated with degenerative arthritis, if handled incorrectly can cause debilitating joint pain and dysfunction.

Mollon et al. (15) observed that the ideal therapeutic treatment for lesions in the synovial joints should involve immobilization and remobilization, so as to restore the properties of support and minimize friction and wear caused by the weight load on this joint. This measure could improve joint mechanics and could delay or even stop the progression of osteoarthritis. Thus, when immobilization becomes crucial, therapeutic measures in order to achieve remobilization (16) can have a positive effect in the articular cartilage and surrounding tissues. 
After a period of immobilization, joint movement exercises provided by remobilization can prevent or reduce stiffness, improve synovial flow and nutrition, and stimulate healing and the regeneration of cartilage (17). Gu et al. (18) have shown that physical exercise can reduce pain, maintain a range of motion, and thus reduce the time of hospitalization or rehabilitation. Among physical exercises, those that are performed in water, which has the added benefits of the physical properties of water such as heat and pressure, can contribute to pain relief and the reduction of edema and can facilitate ease of movement (19). When they are used as resources in various treatments, they can meet most of the objectives proposed in a remobilization program (20).

Considering that immobilization can affect the morphology of the ankle joint, and based on the benefits arising from exercise in water, a protocol composed of a combination of swimming and jumping in water can possibly contribute to a better morphological response of the joint after remobilization.

Consequently, considering the frequent use of immobilization as a therapeutic modality in musculoskeletal injuries, there is a lack of scientific information about its effects on the ankle joint and the large number of residual symptoms, as well as about remobilization of the joint with exercises in water. Therefore, the present study aimed to analyze the effects of immobilization of the ankle joint and the association of swimming and jumping in water on the remobilization of the ankle joint in Wistar rats.

\section{Material and Methods}

\section{Characterization of samples}

Eighteen male Wistar rats, aged $10 \pm 2$ weeks and weighing approximately $280-350 \mathrm{~g}$, were obtained from the Central Animal Laboratory of the Universidade Estadual do Oeste do Paraná (UNIOESTE). The animals were kept in a $12: 12 \mathrm{~h}$ light-dark photoperiod and a temperature of $23 \pm 1{ }^{\circ} \mathrm{C}$, with water and food ad libitum, and there were 3 animals to each box. All animals had their right hindlimb immobilized for 14 days and were divided into 3 experimental groups, with 6 rats in each group as follows: G1, euthanized immediately after the period of immobilization; $\mathrm{G} 2$, free remobilization in the cage for 14 days and, in addition, the animals were placed in contact with water for about 1 min so that they received aquatic stimulus daily; G3, remobilized for 14 days by swimming and jumping in water, performed on alternate days, totaling 7 days of treatment with swimming and 7 days of treatment with jumping.

In this study, as in the study by Baroni et al. (9), the values reported for the left limb served as the control to check the adjustments made to the right member (immobilized/remobilized). The experiment was performed in order to simulate the conditions that normally occur in humans.
All methodological procedures were approved by the Ethics Committee on Animal Use, Cascavel campus, UNIOESTE, PR, Brazil (protocol \#03012).

\section{Immobilization protocol}

An immobilizing apparatus was used, which was made from material suitable for plastering a body segment. It comprised a ligation saturated with dried calcium sulfate $\left(\mathrm{CaSO}_{4}\right)$ in white powder form, characterized as a plaster bandage, according to the model proposed by Carvalho et al. (21). All experimental groups had the described orthosis, which weighed approximately $50 \mathrm{~g}$, molded directly onto the right hindlimb of each animal; it was placed along the length of the limb so that the limb remained extended from the knee joint with complete plantar flexion of the ankle, i.e., in a position of shortening the soleus muscle, which was maintained in the position described above, for a period of 15 consecutive days.

Verification of immobilization was performed daily, and, at the end of the period of immobilization, the presence of edema or skin lesions was not observed.

\section{Remobilization protocol}

The remobilization protocol was performed in a water tank with a capacity of $220 \mathrm{~L}$ and a controlled temperature of $31 \pm 2^{\circ} \mathrm{C}$.

The animals in the G2 group were allowed free remobilization in the cage and were only placed in contact with water. They were housed individually so that the body remained submerged for 1 minute daily for 14 days in order to ensure equal treatment between the experimental groups, minimizing different stress responses by the action of contact with water.

Animals in the G3 group underwent remobilization; they had alternating swimming exercises, simulating the front crawl, and jumping in water for a period of 14 days. These animals were gradually trained for 5 consecutive days before the immobilization period.

During the first 6 days of remobilization, swimming was performed for 20 min and jumps were performed in two sets of 10 jumps, with 30 s of rest between sets, on alternate days. During the remaining 8 days of remobilization, on alternate days, a progression in terms of time and series of exercises was performed; swimming time was 40 min and jumps were performed in four sets of 10 jumps, with $1 \mathrm{~min}$ of rest between sets. The swimming exercises were performed in groups of 3 animals per time and without any additional weight, while the jumping exercises in water were conducted individually and with an additional $50 \%$ of body weight, according to model adjustments described by Gaffuri et al. (22).

\section{Euthanasia of the animals and sample collection}

For monitoring of body weight, all animals were weighed before immobilization and after removal of the orthosis. Animals in groups G2 and G3 were weighed at the end of remobilization. 


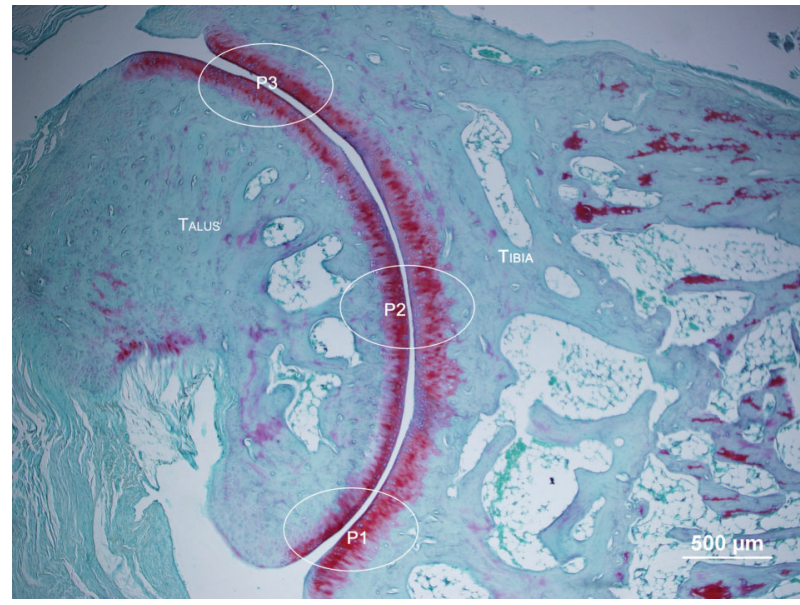

Figure 1. Photomicrograph of the ankle joint, showing the areas of measurement in the joint: P1: anterior articular extremity; P2: mid-region of the joint; P3: posterior articular extremity. Sagittal section, safarin $\mathrm{O}$ fast green staining.

Animals in group G1 (shortly after the period of immobilization) and animals in groups G2 and G3 (after remobilization) were weighed and anesthetized with $50 \mathrm{mg} / \mathrm{kg}$ ketamine hydrochloride and $10 \mathrm{mg} / \mathrm{kg}$ xylazine hydrochloride. The animals were decapitated by guillotine while under the effect of anesthetics. The right ankle joints (of the animals submitted to immobilization/remobilization) and the left ankle joints (control) were subsequently dissected and then cleaned and placed in $10 \%$ formalin for subsequent histological analysis.

\section{Preparation of the slides and histological analysis}

After fixation, the ankle joints were washed in distilled water and decalcified in 5\% trichloroacetic acid for about 5 days, following the protocol for paraffin embedding. Subsequently, microtomy was performed with slices of $7 \mu \mathrm{m}$ using an Olympus CUT 4055 microtomer (Germany). The slides, of a sagittal section of the ankle joint, were stained with hematoxylin and eosin (23) for general morphological tissue analysis, and with safarin $O$ fast green staining (24) for analysis of articular cartilage.

After the slides were prepared, they were examined under a light microscope (Olympus), where the visual fields of interest were photomicrographed using an Olympus DP71 microscope. The protocol for measuring the thickness of the articular cartilage and the number of chondrocytes was adapted from Ando et al. (25) and Hagiwara et al. (26). Three distinct fields of interest were identified (Figure 1): P1, anterior articular extremity (close to the phalanges); P2, midregion of the joint; and $\mathrm{P} 3$, posterior articular extremity.

These areas of interest were photomicrographed at $200 \times$ magnification and the total thickness of the cartilage, defined as the distance between the cartilaginous surface and the osteochondral junction, was measured at 3 points using the Image Pro Plus 6.0 program (USA). In order to count the number of chondrocytes, an area of interest (a rectangle $100 \mu \mathrm{m}$ in depth by $200 \mu \mathrm{m}$ in length) was chosen, which was superimposed on the 3 points of analysis (P1, P2, and P3), where the exclusion borders were considered to be the margins greater than $100 \mu \mathrm{m}$ and deeper than $200 \mu \mathrm{m}$, closest to the subchondral bone.

During analysis of the slides, the morphological characteristics of the synovial membrane were observed, with the anterior articular region as the standard observation point; articular cartilage was analyzed for the presence of flocculation on the articular surface and for cracks and cellular organization; and the articular cavity was analyzed for the presence of synovial infiltration and the formation of pannus.

\section{Statistical analysis}

Morphometric data relating to the tibia and talus, as well as body weight, were analyzed using the SPSS 17.0 program (Statistical Package for the Social Sciences) (USA) and are reported as means $\pm S D$. Analysis of variance was used to analyze the groups (G1, G2, and G3) and the right (immobilized/remobilized) and left (control) sides of the ankle of each group and between groups. The level of significance was $5 \%$.

\section{Results}

In relation to body weight, there was no significant difference between groups in any evaluation. However, there was a reduction in body weight after 14 days of immobilization $(P<0.05)$ in all groups, but animals in $G 2$ and $\mathrm{G} 3$ regained weight after remobilization.

In the histomorphometric analysis of the number of chondrocytes present in the articular cartilage of the tibia and talus, there were no significant differences between the groups or between the control and immobilized/remobilized sides in any group (Table 1). Similarly, no differences were observed in the thickness of the articular cartilage (Table 2).

For all groups, the joint of the left ankle (control) showed a characteristic morphology, with the articular cartilage having a smooth surface and organized into 4 normal cell layers (Figure $2 \mathrm{~A}$ and $\mathrm{B}$ ). In the surface zone, a greater cell density was observed, with chondrocytes arranged in horizontal agglomerations of flattened appearance. In the intermediate zone, the cells had a rounded appearance and were arranged either alone or in isogenous groups. The chondrocytes were organized into gaps that corresponded to the deep zone, separated from the calcified zone by a basophilic line called the tidemark.

The synovial membrane also had normal characteristics, i.e., 2 to 3 layers of cells (synovial type A and type B) in the synovial intima and subintima, with a predominance of adipose cells (Figure $3 \mathrm{~A}$ ).

In 3 animals of the group that was simply immobilized, the cartilage did not have the normal morphological characteristics described for the other contralateral control 
Table 1. Number of chondrocytes in the articular cartilage of the tibia and the talus.

\begin{tabular}{cccc}
\hline & \multicolumn{3}{c}{ Number of chondrocytes } \\
\cline { 2 - 4 } & G1 & G2 & G3 \\
\hline Tibia & & & \\
P1 & & & \\
$\quad$ Right & $30.4 \pm 9.5$ & $31.8 \pm 8.6$ & $35.2 \pm 7.8$ \\
$\quad$ Left & $36.0 \pm 14.1$ & $31.7 \pm 6.8$ & $29.7 \pm 7.5$ \\
P2 & & & \\
$\quad$ Right & $37.8 \pm 6.6$ & $34.0 \pm 5.4$ & $41.2 \pm 7.7$ \\
$\quad$ Left & $36.2 \pm 4.8$ & $39.7 \pm 15.2$ & $34.8 \pm 6.9$ \\
P3 & & & \\
$\quad$ Right & $31.6 \pm 7.4$ & $30.2 \pm 8.6$ & $29.8 \pm 4.2$ \\
Left & $27.7 \pm 5.9$ & $30.8 \pm 7.7$ & $28.3 \pm 9.6$ \\
Talus & & & \\
P1 & & & \\
$\quad$ Right & $36.2 \pm 8.2$ & $28.0 \pm 5.3$ & $38.0 \pm 8.0$ \\
Left & $29.7 \pm 5.4$ & $32.3 \pm 5.5$ & $30.7 \pm 11.8$ \\
P2 & & & \\
$\quad$ Right & $14.8 \pm 8.5$ & $19.8 \pm 8.7$ & $27.7 \pm 7.7$ \\
$\quad$ Left & $26.8 \pm 9.2$ & $32.0 \pm 6.6$ & $26.5 \pm 5.8$ \\
P3 & & & \\
Right & $38.8 \pm 8.1$ & $31.5 \pm 5.5$ & $38.5 \pm 9.4$ \\
Left & $35.3 \pm 8.4$ & $33.5 \pm 7.5$ & $35.7 \pm 9.0$ \\
\hline
\end{tabular}

Data are reported as means \pm SD. G1: immobilized; G2: remobilized freely for 14 days; G3: remobilized by swimming and jumping in water for 14 days; $\mathrm{P} 1$ : anterior articular extremity; P2: mid-region of the joint; P3: posterior articular extremity.

limbs; they had injuries in the articular cartilage between the anterior region and the center of the joint, as well as cracks in the center of the articular cartilage of the tibia. A slight thickening of the synovial membrane was also observed in the animals in group G1 (not shown).

In the right ankle joint of animals in group G1, immobilization caused complete loss of articular cartilage of the talus, between the anterior articular region (P1) and the center of the joint (P2), with the subchondral bone exposed (Figure 2C), which was not observed in the tibia. A slight decrease in intensity of staining with hematoxylin and eosin along the cartilage was also found in the tibia and talus, as well as disappearance and/or discontinuation of the tidemark. Large areas of flocculation and cracks in the deeper layers (Figure 2D) were observed at some points on the surface of the cartilage.

In the G1 group, the synovial membrane was thickened by the presence of blood infiltration, predominantly in the subintima, with an increase in red blood cells within the blood vessels and replacement of the type of connective tissue, from adipose to fibrous (Figure 3B).

Animals in the $\mathrm{G} 2$ group showed regions of destruction of the articular cartilage of the talus, but these regions were fully recovered by pannus, also known as granulation tissue, so that there was no exposure of the subchondral
Table 2. Articular cartilage thickness $(\mu \mathrm{m})$ of the tibia and the talus.

\begin{tabular}{cccc}
\hline & \multicolumn{3}{c}{ Cartilage thickness $(\mu \mathrm{m})$} \\
\cline { 2 - 4 } & G1 & G2 & G3 \\
\hline $\begin{array}{c}\text { Tibia } \\
\text { P1 }\end{array}$ & & & \\
$\quad$ Right & $237.1 \pm 42.9$ & $212.7 \pm 28.8$ & $207.5 \pm 32.1$ \\
$\quad$ Left & $224.8 \pm 28.5$ & $235.1 \pm 18.1$ & $228.5 \pm 41.1$ \\
P2 & & & \\
$\quad$ Right & $231.5 \pm 28.9$ & $186.2 \pm 21.8$ & $196.9 \pm 61.1$ \\
$\quad$ Left & $200.9 \pm 43.3$ & $197.2 \pm 19.2$ & $207.7 \pm 51.4$ \\
P3 & & & \\
$\quad$ Right & $139.6 \pm 42.1$ & $136.8 \pm 71.1$ & $152.3 \pm 46.1$ \\
$\quad$ Left & $144.5 \pm 55.4$ & $157.4 \pm 31.1$ & $124.5 \pm 20.7$ \\
Talus & & & \\
P1 & & & \\
$\quad$ Right & $134.4 \pm 28.1$ & $154.2 \pm 36.0$ & $163.7 \pm 36.6$ \\
$\quad$ Left & $162.2 \pm 23.1$ & $162.0 \pm 27.7$ & $147.5 \pm 16.7$ \\
P2 & & & \\
$\quad$ Right & $132.0 \pm 67.7$ & $122.8 \pm 68.0$ & $145.9 \pm 45.5$ \\
$\quad$ Left & $148.8 \pm 34.2$ & $147.2 \pm 22.7$ & $179.6 \pm 43.2$ \\
P3 & & & \\
$\quad$ Right & $134.5 \pm 35.6$ & $163.8 \pm 70.0$ & $150.9 \pm 37.1$ \\
$\quad$ Left & $139.5 \pm 42.4$ & $154.9 \pm 13.2$ & $150.4 \pm 20.1$ \\
\hline
\end{tabular}

Data are reported as means $\pm \mathrm{SD}$. G1: immobilized; G2 remobilized freely for 14 days; G3: remobilized by swimming and jumping in water for 14 days; P1: anterior articular extremity; P2: mid-region of the joint; P3: posterior articular extremity.

bone (Figure 2E). The presence of cracks and flocculation, mainly in the talus, and discontinuity of the tidemark (Figure $2 \mathrm{~F}$ ) were also noted.

Regarding the synovial membrane of animals in the G2 group, the intima was fibrous and disorganized, and a loss of epithelioid arrangement was verified. In the subintimal region, partial recovery of tissue organization was observed, with the reappearance of adipocytes. Moderate amounts of red blood cells were seen within the blood vessels, although there was blood infiltration in the connective tissue (Figure $3 \mathrm{C}$ ).

In the G3 group, areas of recovery of cartilage were observed by the presence of cell clones (Figure 2G), instead of pannus, covering the subchondral bone. However, there was a persistence of small areas with flocculation and minicracks as well as a mild derangement of the tidemark (Figure $2 \mathrm{H}$ ). In the synovial membrane, the intima showed areas of tissue reorganization, assuming an epithelioid appearance. The subintima was less fibrous, with the presence of fat cells and few red blood cells in the blood vessels (Figure 3D).

\section{Discussion}

Immobilization for 14 days led to a reduction in body weight of the animals in all groups. Mosekilde et al. (27) 

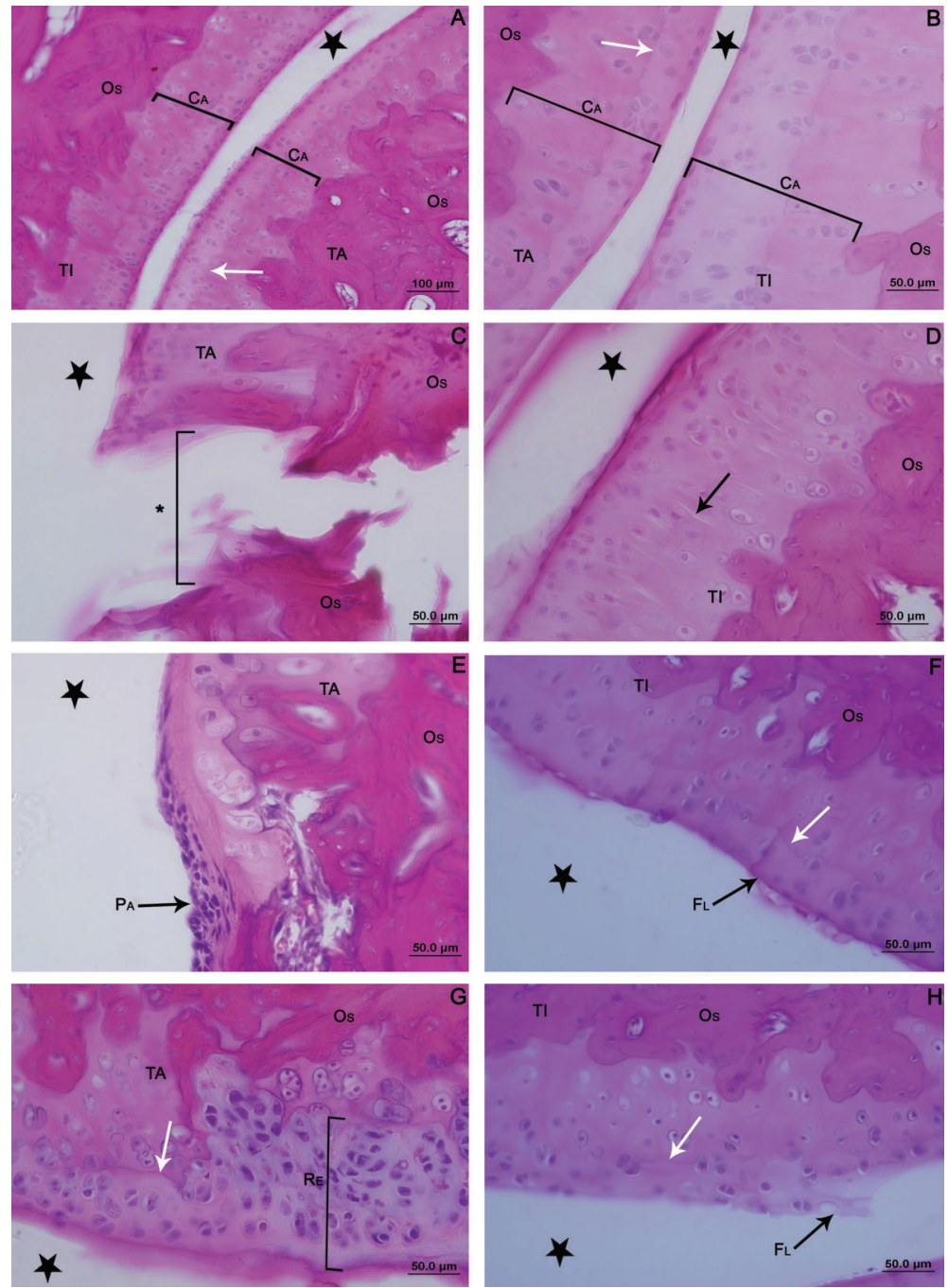

Figure 2. Photomicrographs of the articular cartilage of the ankle joint of the control contralateral hindlimb of Wistar rats $(A$ and $B) ; \mathrm{G} 1$ ( $C$ and $D)$; $\mathrm{G} 2(E$ and $F)$; $\mathrm{G} 3$ ( $G$ and $H)$. Sagittal section, hematoxylin and eosin staining. $A$, Panoramic view showing the articular cartilage (CA). B, Details of cellular organization. $C$, Loss of cartilage (asterisk) with exposed subchondral bone (OS). $D$, Cracks (black arrow). E, Pannus formation (PA). F, Presence of flocculation on the surface of cartilage $(\mathrm{FL})$. G, Recovery of articular cartilage with the presence of clones (RE). $H$, Flocculation on the surface. Articular cavity (star), tidemark (white arrow), talus bone (TA) and tibia ( $\mathrm{Tl})$. G1: immobilized; G2: remobilized freely for 14 days; G3: remobilized by swimming and jumping in water for 14 days. also observed a decrease in body weight in their model of animal immobilization as a result of a decrease in food consumption and lower overall mobility. Nevertheless, it is noteworthy that, after remobilization, the animals in both $\mathrm{G} 2$ and $\mathrm{G} 3$ regained their body weight, demonstrating that immobilization did not result in long-term stress.

Immobilization can lead to different characteristics between the areas of contact, noncontact, and transitional knee joint, such as changes in cartilage thickness, as well as changes in the amount and distribution of chondrocytes and the proteoglycan matrix (24-26). Consequently, histomorphometric analysis of the articular cartilage of the ankle was performed in the anterior, middle, and posterior regions (Figure 1), providing a reference standard for the measurements of the number of chondrocytes and also the cartilage thickness.

Immobilization, as well as therapeutic exercises and free remobilization, did not alter the number of chondrocytes and the thickness of articular cartilage of the ankle during the period of study. Studying the knee, Hagiwara et al. (26) found that, after 2 weeks of immobilization at an angle of $150^{\circ}$ of knee flexion, there was a decrease in the number of chondrocytes in the contact areas of the tibia and the femur, while, in the transitional area, this decrease was observed at 8 and 16 weeks for the femur and tibia, respectively. Similar results were also found by Ando et al. (25).

Thus, even though they are different joints, it seems that an increased period of immobilization is necessary in order to cause a change in the number of chondrocytes in the ankle joint, explaining the difference in results.

Renner et al. (28) and Dias et al. (29) studied the effects of a muscle-stretching protocol after 4 weeks of immobilization of the ankle joint. Although their analysis of cartilage thickness and the number of chondrocytes was conducted in different regions, the authors found no differences in the thickness of the articular cartilage between the groups, as in the present study. Regarding the number of chondrocytes, 

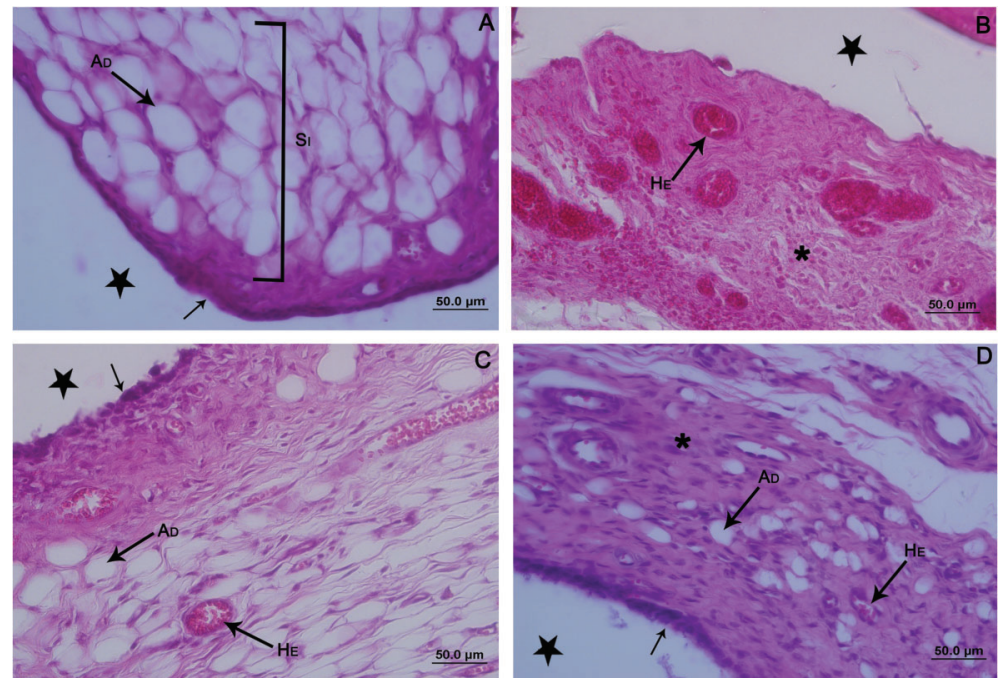

Figure 3. Photomicrographs of the synovial membrane of the ankle joint of Wistar rats. Sagittal section, hematoxylin and eosin staining. A: Control contralateral hindlimb. Membrane with thin synovial intima (black arrow), and subintima (SI) with predominance of fat cells (AD). B, G1 group, thickening of the synovial membrane, which was predominantly fibrous (asterisk) and with blood vessels (HE) full of red blood cells that spilled over the connective tissue. C, G2 group, synovial membrane with thickening in the apical region and intima with disorganized synoviocytes (black arrow). Presence of adipocytes (AD) in the subintima, with a moderate amount of red blood cells within the blood vessels (HE). D, G3 group, synovial membrane with areas of reorganization of the intima (black arrow). Subintima less fibrous (asterisk), with fat cells $(A D)$ and few red blood cells in the blood vessels (HE). Articular cavity (star). G1: immobilized; G2: remobilized freely for 14 days; G3: remobilized by swimming and jumping in water for 14 days. the authors found that there was an increase in the number of cells of the animals subjected to the stretching protocol after immobilization.

Although there are no studies regarding the ankle joint, studies of the knee, in terms of immobilization, have variously observed an increase in thickness (26), or no change (24-25). Thus, different methods of immobilization, angles of joint fixation (extension or flexion), and various areas of study hamper consensus within the literature about the effects of immobilization on the thickness of articular cartilage.

In the present study, immobilization caused more significant degenerative changes in the articular cartilage of the talus compared to the tibia. Vanwanseele et al. (30) stated that the functional demands, to which the cartilage in the synovial joints are subjected, are responsible for the morphology of articular cartilage and the maintenance of its homeostasis. Therefore, it can be inferred that the absence of load, due to the immobilization of the joint for 15 days, seems to have been the cause of the morphological changes in the articular cartilage of the talus.

Furthermore, fixed immobilization of the ankle joint caused complete destruction of the articular cartilage between the front region (P1) and the center (P2) of the joint. According to Del Carlo et al. (31), this type of immobilization may hinder the diffusion of synovial fluid in the joint cavity, decreasing the supply of nutrients to the chondrocytes, which respond to changes induced by disuse, with decreased secretion of extracellular matrix and proteoglycan synthesis (30). Nagase and Kashiwagi (32) stated that degradation of the extracellular matrix and complete erosion of articular cartilage may be caused by increased activity of proteolytic enzymes, known as matrix metalloproteinases.

The imbalance in the distribution of forces on the joint surface caused less pronounced degenerative changes in the other regions of the cartilage (center P2 and posterior region $\mathrm{P} 3$ ), such as a rupture in the arrangement of the proteoglycans, the appearance of flocculation on the surface, and cracks in the deeper layers, as also observed in the knee joint by Melo et al. (33).

The less intense staining to the articular cartilage after immobilization of the ankle may be due to decreased synthesis of the extracellular matrix, as noted by Ando et al. (24), in their studies of the knee.

It is worth noting that the lesion between the anterior region and the center of the joint of the contralateral control hindlimb in 3 of the immobilized animals (G1) can be explained by the greater use of the left limb in locomotion during the period of immobilization of the right limb. Thus, both the excess load exerted on the cartilage, as occurred on the left side, and the absence of this on the immobilized side, can lead to adaptations in the articular cartilage. Previous studies have shown a change in the composition of cartilage matrix (34) and also the mechanical properties of cartilage (35) on the contralateral side.

In the case of changes in the synovial membrane of the immobilized animals (G1), Del Carlo et al. (31) found that immobilization leads to stiffness of the joint, with a consequent decreased range of motion, causing a thickening of the synovial membrane; this result was also found by Melo et al. (33), Ando et al. (36), and Trudel et al. (37).

In the case of animals that were freely remobilized (G2), the presence of pannus, covering the damaged regions of cartilage, resulted in inflammatory phenomena resulting from intra- and periarticular tissues (33). Fassbender (38) found that proliferation of mesenchymal cells originating from the synovial membrane preceded the formation of pannus, which then adhered to the articular cartilage. Allard et al. (39) stated that pannus may induce articular cartilage damage by the production of proteolytic enzymes and the 
interaction of cytokines with cells from the cartilage and the subchondral bone. In addition, Ando et al. (36) suggested that immobilization causes changes in the secretion and distribution of hyaluronic acid, leading to the conversion of immature adhesive tissue into mature tissue, and affecting the adhesive property of the extracellular matrix, causing deposition of pannus over the articular cartilage.

In the present study, pannus formation only occurred in regions where the subchondral bone was exposed. Thus, in accordance with Del Carlo et al. (31), the presence of pannus in this area seems to reflect a healing reaction in response to the damage caused by the destruction of cartilage and, associated with the medullary blood vessels in the region, it meets the nutritional and repair requirements of the subchondral bone.

It was found that, in both the animals that were freely remobilized (G2) and those that were subjected to therapeutic exercise (G3), the synovial membrane showed an intermediate aspect, compared to the control contralateral limbs and the animals that were only immobilized (G1). Del Carlo et al. (31) also observed that both free remobilization, as well as swimming, positively influenced the morphological return of the synovial membrane in the knee joint. Nevertheless, in the present study, it was observed that the exercises performed by the animals in group G3 seem to have had a more significant reparative effect, evidenced by the improved epithelioid arrangement of the synovial intima, decreased infiltration of blood, and the return of normal connective properties of the subintima.

The combination of therapeutic exercises (swimming and jumping) aided the restoration of movement, improved the flow of synovial fluid in the joint cavity, and aided the

\section{References}

1. Junge A, Engebretsen L, Mountjoy ML, Alonso JM, Renstrom PA, Aubry MJ, et al. Sports injuries during the Summer Olympic Games 2008. Am J Sports Med 2009; 37: 2165-2172, doi: 10.1177/0363546509339357.

2. Katcherian DA. Soft-tissue injuries of the ankle. In: Lutter LD, Mizel MS, Pfeffer GB (Editors), Orthopaedic knowledge update: foot and ankle. Rosemont: American Academy of Orthopaedic Surgeons; 1994. p 241-253.

3. Holmer P, Sondergaard L, Konradsen L, Nielsen PT, Jorgensen LN. Epidemiology of sprains in the lateral ankle and foot. Foot Ankle Int 1994; 15: 72-74, doi: 10.1177/10711 0079401500204.

4. Belangero PS, Tamaoki MJS, Nakama GY, Shoiti MV, Gomes RVF, Belloti JC. How does the Brazilian orthopedic surgeon treat acute lateral ankle sprain? Rev Bras Ortop 2010; 45: 468473, doi: 10.1590/S0102-36162010000500015.

5. Kerkhoffs GM, Rowe BH, Assendelft WJ, Kelly K, Struijs $\mathrm{PA}$, van Dijk CN. Immobilisation and functional treatment for acute lateral ankle ligament injuries in adults. Cochrane Database Syst Rev 2002; CD003762.

6. van Rijn RM, van Os AG, Bernsen RM, Luijsterburg PA, Koes BW, Bierma-Zeinstra SM. What is the clinical course of acute nutrition of the cartilage, providing regeneration, as described by Ando et al. (25).

According to Melo et al. (33), the presence of cell clones, replacing pannus in articular cartilage (G3), seems to be an attempt to repair the tissue, due to the continuation of the proliferative capacity of chondrocytes, even after periods of immobilization. Del Carlo et al. (31) found that cartilage lesions can be reversed with remobilization programs. In this same study, the authors also confirmed that swimming led to a decrease in joint stress, improving the diffusion of synovial fluid and promoting less severe lesions on the cartilage.

In the present study, the aquatic exercises used in group $\mathrm{G} 3$ resulted in a more efficient recovery of the ankle cartilage compared with free remobilization (G2). This can be attributed to a lower gravitational force, which facilitates joint movement and, consequently, the distribution of the synovial liquid required to maintain homeostasis. Zaldivar et al. (40) further argued that physical exercise increases the number of circulating pro- or anti-inflammatory cells, such as interleukins, which are responsible for tissue regeneration and repair.

In conclusion, it was found that immobilization of the ankle joint caused degeneration of articular cartilage, mainly in the talus, and changes in the synovial membrane. Regarding remobilization by therapeutic exercises in water, there was an improvement in the morphological organization of the ankle joint, both in the cartilage and in the synovial membrane, when compared with free remobilization. Studies over longer time periods and other therapeutic modalities would be beneficial to confirm the positive effects of remobilization of the ankle joint. ankle sprains? A systematic literature review. Am J Med 2008; 121: 324-331, doi: 10.1016/j.amjmed.2007.11.018.

7. Gerber JP, Williams GN, Scoville CR, Arciero RA, Taylor DC. Persistent disability associated with ankle sprains: a prospective examination of an athletic population. Foot Ankle Int 1998; 19: 653-660, doi: 10.1177/1071100798019 01002.

8. Kerkhoffs GM, Struijs PA, Marti RK, Assendelft WJ, Blankevoort L, van Dijk CN. Different functional treatment strategies for acute lateral ankle ligament injuries in adults. Cochrane Database Syst Rev 2002; CD002938.

9. Baroni BM, Galvão AQ, Ritzel CH, Diefenthaeler F, Vaz MA. Dorsiflexor and plantarflexor neuromuscular adaptations at two-week immobilization after ankle sprain. Rev Bras Med Esporte 2010; 16: 358-362, doi: 10.1590/S1517-86922010000 500008.

10. Lamb SE, Marsh JL, Hutton JL, Nakash R, Cooke MW. Mechanical supports for acute, severe ankle sprain: a pragmatic, multicentre, randomised controlled trial. Lancet 2009; 373: 575-581, doi: 10.1016/S0140-6736(09)60206-3.

11. Arakaki K, Kitamura N, Kurokawa T, Onodera S, Kanaya F, Gong JP, et al. Joint immobilization inhibits spontaneous 
hyaline cartilage regeneration induced by a novel doublenetwork gel implantation. J Mater Sci Mater Med 2011; 22: 417-425, doi: 10.1007/s10856-010-4216-0.

12. Thaxter TH, Mann RA, Anderson CE. Degeneration of immobilized knee joints in rats. J Bone Joint Surg Am 1965; 47: 567-585.

13. Widuchowski W, Lukasik P, Kwiatkowski G, Faltus R, Szyluk $\mathrm{K}$, Widuchowski $\mathrm{J}$, et al. Isolated full thickness chondral injuries. Prevalance and outcome of treatment. A retrospective study of 5233 knee arthroscopies. Acta Chir Orthop Traumatol Cech 2008; 75: 382-386.

14. Bedi A, Feeley BT, Williams RJ III. Management of articular cartilage defects of the knee. J Bone Joint Surg Am 2010; 92: 994-1009, doi: 10.2106/JBJS.I.00895.

15. Mollon B, Kandel R, Chahal J, Theodoropoulos J. The clinical status of cartilage tissue regeneration in humans. Osteoarthritis Cartilage 2013; 21: 1824-1833, doi: 10.1016/ j.joca.2013.08.024.

16. Simon TM, Jackson DW. Articular cartilage: injury pathways and treatment options. Sports Med Arthrosc 2006; 14: 146154, doi: 10.1097/00132585-200609000-00006.

17. Salter RB. History of rest and motion and the scientific basis for early continuous passive motion. Hand Clin 1996; 12: 1-11.

18. Gu XI, Leong DJ, Guzman F, Mahamud R, Li YH, Majeska $\mathrm{RJ}$, et al. Development and validation of a motion and loading system for a rat knee joint in vivo. Ann Biomed Eng 2010; 38: 621-631, doi: 10.1007/s10439-009-9865-0.

19. Hinman RS, Heywood SE, Day AR. Aquatic physical therapy for hip and knee osteoarthritis: results of a singleblind randomized controlled trial. Phys Ther 2007; 87: 3243, doi: 10.2522/ptj.20060006.

20. Resende SM, Rassi CM, Viana FP. Effects of hydrotherapy in balance and prevention of falls among elderly women. Rev Bras Fisioter 2008; 12: 57-63, doi: 10.1590/S1413-35552008 000100011.

21. Carvalho CMM, Shimano AC, Volpon JB. Effects of immobilization and physical training on the mechanical properties of the striated muscle. Rev Bras Eng Biomed 2002; 18: 65-73.

22. Gaffuri J, Meireles A, Rocha BP, Rosa CT, Artifon EL, Silva LI, et al. Physical exercise assessment as na analgesia factor in a sciatica experimental model. Rev Bras Med Esporte 2011; 17: 115-118, doi: 10.1590/S1517-86922011000200009.

23. Junqueira LC, Junqueira LMMS. Técnicas básicas de citologia e histologia. São Paulo: Santos; 1983.

24. Ando A, Hagiwara $\mathrm{Y}$, Chimoto $\mathrm{E}$, Hatori K, Onoda $\mathrm{Y}$, Itoi E. Intra-articular injection of hyaluronan diminishes loss of chondrocytes in a rat immobilized-knee model. Tohoku $\mathrm{J}$ Exp Med 2008; 215: 321-331, doi: 10.1620/tjem.215.321.

25. Ando A, Suda H, Hagiwara Y, Onoda Y, Chimoto E, Saijo Y, et al. Reversibility of immobilization-induced articular cartilage degeneration after remobilization in rat knee joints. Tohoku $\mathrm{J}$ Exp Med 2011; 224: 77-85, doi: 10.1620/tjem.224.77.

26. Hagiwara $Y$, Ando A, Chimoto E, Saijo Y, Ohmori-Matsuda K, Itoi $\mathrm{E}$. Changes of articular cartilage after immobilization in a rat knee contracture model. J Orthop Res 2009; 27: 236-242, doi: 10.1002/jor.20724.

27. Mosekilde L, Thomsen JS, Mackey MS, Phipps RJ. Treatment with risedronate or alendronate prevents hindlimb immobilization-induced loss of bone density and strength in adult female rats. Bone 2000; 27: 639-645, doi: 10.1016/S8756-3282(00)00375-6.

28. Renner AF, Carvalho E, Soares E, Mattiello-Rosa S. The effect of a passive muscle stretching protocol on the articular cartilage. Osteoarthritis Cartilage 2006; 14: 196-202, doi: 10.1016/j.joca.2005.08.011.

29. Dias CN, Renner AF, dos Santos AA, Vasilceac FA, Mattiello SM. Progression of articular cartilage degeneration after application of muscle stretch. Connect Tissue Res 2012; 53: 39-47, doi: 10.3109/03008207.2011.610476.

30. Vanwanseele B, Lucchinetti E, Stussi E. The effects of immobilization on the characteristics of articular cartilage: current concepts and future directions. Osteoarthritis Cartilage 2002; 10: 408-419, doi: 10.1053/joca.2002.0529.

31. Del Carlo RJ, Galvão MR, Viloria MIV, Natali AJ, Barbosa ALT, Monteiro BS, et al. Experimental immobilization and remobilization rat knee joints: clinical and microscopic study. Arq Bras Med Vet Zootec 2007; 59: 363-370, doi: 10.1590/ S0102-09352007000200015.

32. Nagase $H$, Kashiwagi $M$. Aggrecanases and cartilage matrix degradation. Arthritis Res Ther 2003; 5: 94-103, doi: 10.1186/ ar630.

33. Melo EG, Nunes VA, Rezende CMF, Gomes MG, Malm C, Gheller VA. Chondroitin sulfate and sodium hyaluronate in the treatment of the degenerative joint disease in dogs. Histological features of articular cartilage and synovium. Arq Bras Med Vet Zootec 2008; 60: 83-92, doi: 10.1590/S010209352008000100013

34. Setton LA, Mow VC, Muller FJ, Pita JC, Howell DS. Mechanical behavior and biochemical composition of canine knee cartilage following periods of joint disuse and disuse with remobilization. Osteoarthritis Cartilage 1997; 5: 1-16, doi: 10.1016/S1063-4584(97)80027-1.

35. Leroux MA, Cheung HS, Bau JL, Wang JY, Howell DS, Setton LA. Altered mechanics and histomorphometry of canine tibial cartilage following joint immobilization. Osteoarthritis Cartilage 2001; 9: 633-640, doi: 10.1053/joca.2001.0432.

36. Ando A, Hagiwara Y, Onoda Y, Hatori K, Suda H, Chimoto E, et al. Distribution of type $A$ and $B$ synoviocytes in the adhesive and shortened synovial membrane during immobilization of the knee joint in rats. Tohoku J Exp Med 2010; 221: 161-168, doi: 10.1620/tjem.221.161.

37. Trudel G, Jabi M, Uhthoff HK. Localized and adaptive synoviocyte proliferation characteristics in rat knee joint contractures secondary to immobility. Arch Phys Med Rehabil 2003; 84: 1350-1356, doi: 10.1016/S0003-9993(03) 00233-8.

38. Fassbender HG. Histomorphological basis of articular cartilage destruction in rheumatoid arthritis. Coll Relat Res 1983; 3: 141-155, doi: 10.1016/S0174-173X(83)80040-5.

39. Allard SA, Bayliss MT, Maini RN. The synovium-cartilage junction of the normal human knee. Implications for joint destruction and repair. Arthritis Rheum 1990; 33: 11701179, doi: 10.1002/art.1780330818.

40. Zaldivar F, Wang-Rodriguez J, Nemet D, Schwindt C, Galassetti P, Mills PJ, et al. Constitutive pro- and antiinflammatory cytokine and growth factor response to exercise in leukocytes. J Appl Physiol 2006; 100: 1124-1133, doi: 10.1152/japplphysiol.00562.2005. 